\title{
Análise epidemiológica das parasitoses intestinais em escolares de 7 a 12 anos
}

\author{
Epidemiological analysis of intestinal parasites in schoolchildren aged 7 to 12 years
}

Análisis epidemiológico de las parasitosis intestinal en escolares de 7 a 12 años

Janniele de Souza Cruz ${ }^{1}$, Sannara Inácia dos Santos ${ }^{1}$, Kécya Patricia Costa Macêdo ${ }^{1}$, Janaina Vidal Bezerra $^{1}$, Eduardo Sousa de Araújo Costa ${ }^{2}$, Grasielly Menezes Santos ${ }^{8}$, Francisco Itallo Soares Azevedo $^{7}$, Amanda da Costa Andrade ${ }^{2}$, Jonh Keuvin Andrade de Oliveira ${ }^{8}$, Renandro de Carvalho Reis $^{2}$, Raquel Morais da Silva ${ }^{9}$, Roseane Mara Cardoso Lima Verde ${ }^{3}$, Francisco das Chagas Araújo Sousa ${ }^{4}$, Evaldo Hipólito de Oliveira ${ }^{2 *}$, Antônio Carlos de Carvalho ${ }^{1}$.

\section{RESUMO}

Objetivo: Analisar a prevalência de parasitas intestinais em escolares de 7 a 12 anos em Teresina-PI. Métodos: Este estudo baseou-se em uma estratégia qualitativa e quantitativa de pesquisa de caráter descritivo prospectivo, por meio de uma pesquisa de campo com crianças escolares de 7 a 12 anos. 0 presente trabalho realizou-se em uma escola pública e uma privada. A coleta das amostras fecais ocorreu logo após a aprovação do comitê de ética. Foram entregues as crianças o termo de consentimento livre e esclarecido, os frascos coletores e o questionário socioeconômico, ambiental e cultural para posteriormente serem entregues e devidamente preenchidos pelos pais ou responsáveis. Recebeu-se no prazo de 24 horas após a coleta as amostras refrigeradas, totalizando 44 amostras fecais, cada frasco recebido estava devidamente etiquetado. Resultados: Dos 44 indivíduos que entregaram as amostras fecais, $18(40,9 \%)$ das crianças foram da escola pública e $26(59,0 \%)$ crianças da escola particular, de ambos os sexos com idade entre 7 a 12 anos. Conclusão: As altas prevalências de parasitoses intestinais ainda presentes no Brasil podem ser explicadas em parte, pelos altos custos para melhoria da infraestrutura e saneamento básico.

Palavras-chave: Parasitas intestinais, Prevalência de parasitas intestinais, Exame parasitológico de fezes.

\section{ABSTRACT}

Objective: To analyze the prevalence of intestinal parasites in students ranging from 7 to 12 years old in Teresina, Piauí. Methodology: This study was based upon a qualitative and quantitative strategy of prospective descriptive character by means of a field research with realized on children with school ages ranging from 7 to 12 years. The present work was realized on one public school and one private shcool. The stool data collect took place soon after the Ethics Comittee approval. The children received the consent form, the stool containers and the socioeconomic, enviromental and cultural questionnaire that were meant to be delivered and filled out by their parents. The refrigerated samples were received within 24 hours, totalling 44

${ }^{1}$ Centro Universitário Santo Agostinho. Teresina - PI.

2Universidade Federal do Piauí. Teresina - PI. *E-mail: evaldohipolito@gmail.com

3Universidade Brasil, São Paulo - SP.

${ }^{4}$ Universidade Estadual do Piauí. Faculdade de Ciências Médicas. Teresina - PI.

${ }^{5}$ Faculdade de ensino superior de Floriano. Floriano - PI.

${ }^{6}$ Faculdade Integral Diferencial (FACID). Teresina - PI.

${ }^{7}$ Centro Universitário Uninovafapi. Teresina - PI.

${ }^{8}$ Centro de Educação Tecnológica de Teresina (CET). Teresina - PI.

${ }^{9}$ Associação de Ensino Superior do Piauí (AESPI). Teresina - PI. 
stool samples, each one of them properly identified. Results: From the 44 individuals who delivered the stool samples, $18(41 \%)$ of the children were from the public school and $26(59 \%)$ of them were from the private school, from both sexes and ages ranging from 7 to 12 years old. Conclusion: The high prevalence of intestinal parasitosis still present on Brazil might be partly explained by the high costs of the infrastructural and basic sanitation improvements.

Keywords: Intestinal parasites, Intestinal parasites prevalence, Stool test.

\section{RESUMEN}

Objetivo: Analizar la prevalencia de parásitos intestinales en escolares de 7 a 12 años en Teresina-PI. Métodos: Este estudio se basó en una estrategia cualitativa y cuantitativa de investigación de carácter descriptivo prospectivo, a través de una investigación de campo con niños escolares de 7 a 12 años. El presente trabajo se realizó en una escuela pública y privada. La recolección de las muestras fecales ocurrió poco después de la aprobación del comité de ética. Se entregó a los niños el término de consentimiento libre y esclarecido, los frascos recolectores y el cuestionario socioeconómico, ambiental y cultural para posteriormente ser entregados y debidamente cumplimentados por los padres o responsables. Se recibieron en el plazo de 24 horas después de la recolección las muestras refrigeradas, totalizando 44 muestras fecales, cada frasco recibido estaba debidamente etiquetado. Resultados: De los 44 individuos que entregaron las muestras fecales, $18(40,9 \%)$ de los niños fueron de la escuela pública y 26 (59,0\%) niños de la escuela particular, de ambos sexos con edad entre 7 a 12 años. Conclusión: Las altas prevalencias de parasitosis intestinales todavía presentes en Brasil pueden ser explicadas en parte, por los altos costos para la mejora de la infraestructura y saneamiento básico.

Palabras clave: Parásitos intestinales, Prevalencia de parásitos intestinales, Examen parasitológico de heces.

\section{INTRODUÇÃO}

Nos países em progresso a incidência e a prevalência de parasitas intestinais são mais comprometedoras, pois nesses países estão localizados os piores índices socioeconômicos. Os elevados indicies de parasitos intestinais, é apontada como fator de morbidade em crianças, correspondente aos sinais de infestação pelos parasitos como debilidade mental, nutricional e físico (MACCHIONI F, et al., 2015).

A incidência de parasitoses intestinais está associada às desigualdades sócias econômicas, principalmente na oferta de saneamento básico. Acomete regiões heterogêneas, independentemente de estar situado em centros urbanos ou rurais, assim como, nas diversas faixas etárias, constituindo um indicador de incolumidade e de saúde pública de uma região (SANTOS SM, et al., 2015; SILVA JRCB e BARBOSA TJP, 2011; FERNANDES S, et al., 2011; BELLIN M e GRAZZIOTIN NA, 2011; LODO M, et al., 2010). De acordo com Organização Mundial de Saúde (OMS) no ano de 2017 a carência de instalações sanitárias básicas, como banheiros, é vivenciada por 2,4 bilhões de pessoas e mais de 660 milhões ainda fazem consumo de água de fontes com baixa qualidade.

Segundo a Organização Mundial de Saúde, avalia que no mundo 1,221 bilhão de indivíduos estejam infectados por Ascaris lumbricoides, 795 milhões por Trichuris trichiura, 740 milhões por Ancilostomídeos, cerca de 200 milhões pelo complexo Entamoeba histolytica/Entamoeba dispar e 400 milhões por Giardia lamblia (BORGES WF, et al., 2011).

De acordo com Sousa CDSS, et al. (2015), os parasitos intestinais mais frequentemente encontrados em seres humanos são: Ascaris lumbricoides, Trichuris trichiura e os ancilostomídeos, Necator americanus e Ancylostoma duodenale. Entre os protozoários destacam-se a Entamoeba histolytica e a Giardia intestinalis. Diante disso o objetivo desse estudo é analisar a prevalência de parasitas intestinais em crianças de 7 a 12 anos em Teresina-PI. 


\section{MÉTODOS}

Este estudo baseou-se em uma estratégia qualitativa e quantitativa de pesquisa de caráter descritivo prospectivo, por meio de uma pesquisa de campo com crianças escolares de 7 a 12 anos. Foi realizada em uma escola pública e outra privada no município de Teresina-PI. A coleta das amostras fecais ocorreu logo após a aprovação do comitê de ética, em seguida foi solicitada uma autorização da diretoria de ambas as escolas para falar com as crianças enquanto estavam em horário de aula, em seguida entregou-se o questionário socioeconômico, ambiental e cultural elaborado pelos próprios pesquisadores, para posteriormente serem entregues e devidamente preenchidos pelos pais ou responsáveis.

As variáveis analisadas foram: sexo, idade, dados socioculturais, local onde vivem tipo de piso, se possui animais na residência e dentre outros que constam no questionário. Juntamente com o questionário entregouse o termo de consentimento livre e esclarecido para assinatura dos pais ou responsáveis, os frascos coletores e um informativo com orientações da coleta.

Os pais e/ou responsáveis foram informados quanto à confidencialidade dos dados fornecidos, que sua participação não era obrigatória e que em qualquer etapa da pesquisa eles poderiam abandoná-la. Informando também que seus dados não seriam expostos sob nenhum aspecto.

Cada frasco recebido estava devidamente etiquetado com o nome, sexo e idade da criança. Após a coleta, que foi realizada em casa, o pai ou responsável levou-se o frasco contendo as fezes, juntamente com o Termo de Consentimento Livre e Esclarecido assinado e o questionário socioeconômico devidamente respondido. Combinou-se o prazo para a entrega e recolhimento das amostras com a direção de cada escola. Recebeuse no prazo de 24 horas após a coleta as amostras refrigeradas, totalizando 44 amostras fecais.

Em seguida, encaminhou-se ao Laboratório de Análises Clínicas do Centro Universitário Santo Agostinho dentro de uma caixa de isopor térmica com gelo isotérmico. Esta pesquisa foi submetida ao comitê de ética, pois de acordo com a Resolução 466/2012 do Conselho Nacional de Saúde (CNS) todo e qualquer trabalho que envolva seres humanos de forma direta ou indireta, em sua totalidade ou partes dele, deverá ser submetido a fim de proteger o sujeito da pesquisa, as instituições de pesquisa e até mesmo o próprio pesquisador. Ela foi aprovada na data de 21 de novembro de 2018, dados do parecer: $3.029 .236, n^{\circ}$ do CAAE: 94824818.3.0000.5602.

\section{RESULTADOS}

O presente estudo identificou que a renda familiar dos pais ou responsáveis da escola pública corresponderam a uma elevada porcentagem de $72,2 \%$ em relação à escola particular $30,7 \%$ a 1 salário mínimo.

Em contrapartida de 2 a 3 salários mínimos a escola pública apresentou 27,7\% enquanto a escola particular apresentou 34,6\%. Apenas a escola particular apresentou uma média de $34,6 \%$ em relação a 4 salários mínimos por família.

Diante do exposto pode-se analisar que as famílias de baixa renda são as mais prejudicadas devido aos parasitas, em razão das localidades de moradias, más condições sanitárias o que ocasiona altos índices de prevalência de parasitas.

O simples hábito de lavar as mãos com sabão e água corrente, principalmente, antes de se alimentar, preparar os alimentos ou após o uso do banheiro já previne boa parte das parasitoses intestinais. Com base no questionário aplicado observou-se uma média de $55,5 \%$ na escola pública referente ao hábito de lavar as mãos comparadas a escola particular $38,4 \%$.

Dos 44 indivíduos que entregaram as amostras fecais, 18 (40,9\%) das crianças foram da escola pública e $26(59,0 \%)$ crianças da escola particular, de ambos os sexos com idade entre 7 a 12 anos.

Na Tabela 1 são apresentados os resultados do questionário socioeconômicos das escolas envolvidas de cada participante. 
Tabela 1 - Questionário socioeconômico, ambiental, cultural e comportamental.

\begin{tabular}{|c|c|c|c|c|}
\hline \multirow{2}{*}{ Variável } & \multicolumn{2}{|c|}{ Pública } & \multicolumn{2}{|c|}{ Privada } \\
\hline & $\mathbf{N}$ & $\%$ & $\mathbf{N}$ & $\%$ \\
\hline \multicolumn{5}{|l|}{ Gênero } \\
\hline Feminino & 9 & $50 \%$ & 13 & $50 \%$ \\
\hline Masculino & 9 & $50 \%$ & 13 & $50 \%$ \\
\hline \multicolumn{5}{|l|}{ Idade } \\
\hline 7 anos & 7 & $38,9 \%$ & 9 & $34,6 \%$ \\
\hline 8 anos & 4 & $22,2 \%$ & 7 & $26,9 \%$ \\
\hline 9 anos & 4 & $22,2 \%$ & 2 & $7,7 \%$ \\
\hline 10 anos & 1 & $5,6 \%$ & 3 & $11,5 \%$ \\
\hline 11 anos & 1 & $5,6 \%$ & 2 & $7,7 \%$ \\
\hline 12 anos & 1 & $5,6 \%$ & 3 & $11,5 \%$ \\
\hline \multicolumn{5}{|l|}{ Renda familiar } \\
\hline Até 1 salário & 13 & $72 \%$ & 8 & $30,8 \%$ \\
\hline De 2 a 3 salários & 5 & $18 \%$ & 9 & $34,6 \%$ \\
\hline A partir de 4 salários & 0 & 0 & 9 & $34,6 \%$ \\
\hline \multicolumn{5}{|l|}{ Local onde vive } \\
\hline Área urbana & 18 & $100 \%$ & 26 & $100 \%$ \\
\hline Área rural & 0 & 0 & 0 & 0 \\
\hline \multicolumn{5}{|l|}{ Possui animais } \\
\hline Sim & 7 & $38,9 \%$ & 4 & $15,4 \%$ \\
\hline Não & 11 & $61,1 \%$ & 22 & $84,6 \%$ \\
\hline \multicolumn{5}{|l|}{ Água para consumo } \\
\hline Torneira & 18 & $100 \%$ & 26 & $100 \%$ \\
\hline Mineral & 0 & 0 & 0 & 0 \\
\hline Outra & 0 & 0 & 0 & 0 \\
\hline \multicolumn{5}{|c|}{ Forma de tratamento da água } \\
\hline Filtrada & 17 & $94,4 \%$ & 19 & $73 \%$ \\
\hline Clorada & 1 & $5,6 \%$ & 7 & $27 \%$ \\
\hline \multicolumn{5}{|c|}{ Hábito de brincar na terra } \\
\hline Sim & 18 & $100 \%$ & 8 & $30,7 \%$ \\
\hline Não & 0 & 0 & 18 & $69,3 \%$ \\
\hline \multicolumn{5}{|c|}{ Lavar as mãos antes das refeições } \\
\hline Não & 10 & $55,6 \%$ & 10 & $38,5 \%$ \\
\hline Sim & 2 & $11,1 \%$ & 10 & $38,5 \%$ \\
\hline Às vezes & 6 & $33,3 \%$ & 6 & $23 \%$ \\
\hline \multicolumn{5}{|c|}{ Lavar as mãos após o uso do banheiro } \\
\hline Não & 0 & 0 & 0 & 0 \\
\hline Sim & 2 & $11,1 \%$ & 7 & 26,9 \\
\hline Às vezes & 16 & $88,9 \%$ & 21 & 73,1 \\
\hline \multicolumn{5}{|c|}{ Procedentes de frutas e verduras } \\
\hline Supermercado & 4 & $22.2 \%$ & 17 & $65,4 \%$ \\
\hline Feira municipal & 14 & $77,8 \%$ & 9 & $34,6 \%$ \\
\hline \multicolumn{5}{|c|}{ Destino dos dejetos (fezes) em fossa? } \\
\hline Não & 0 & 0 & 0 & 0 \\
\hline Sim & 18 & $100 \%$ & 26 & $100 \%$ \\
\hline \multicolumn{5}{|c|}{ Realiza exame parasitológico de fezes? } \\
\hline Não & 16 & $88,9 \%$ & 10 & $38,5 \%$ \\
\hline Sim & 2 & $11,1 \%$ & 16 & $61,5 \%$ \\
\hline \multicolumn{5}{|l|}{ Tipo de piso da casa } \\
\hline Piso bruto & 8 & $44,5 \%$ & 0 & 0 \\
\hline Cerâmica & 10 & $55,5 \%$ & $100 \%$ & $100 \%$ \\
\hline
\end{tabular}

Fonte: Cruz JS, et al., 2019.

Através do questionário aplicado, observou que mais da metade $88,8 \%$ das crianças da escola pública não realizaram exame parasitológico de fezes comparado à escola privada $38,4 \%$. Neste caso é sempre 
importante um diagnóstico preciso, através do uso de técnicas sensíveis e específicas, pois o diagnóstico laboratorial constitui um processo importante e essencial no mecanismo de controle e combate às infecções parasitárias intestinais. Tais dados possuem relação com a Figura 1. Nela é possível observar que a frequência de exames parasitológicos negativo é maior na escola privada. Porém, a escola privada ainda possui maior frequência de multiparasitismo.

Figura 1 - Frequência da positividade dos exames parasitológicos de fezes entre as escolas analisadas.

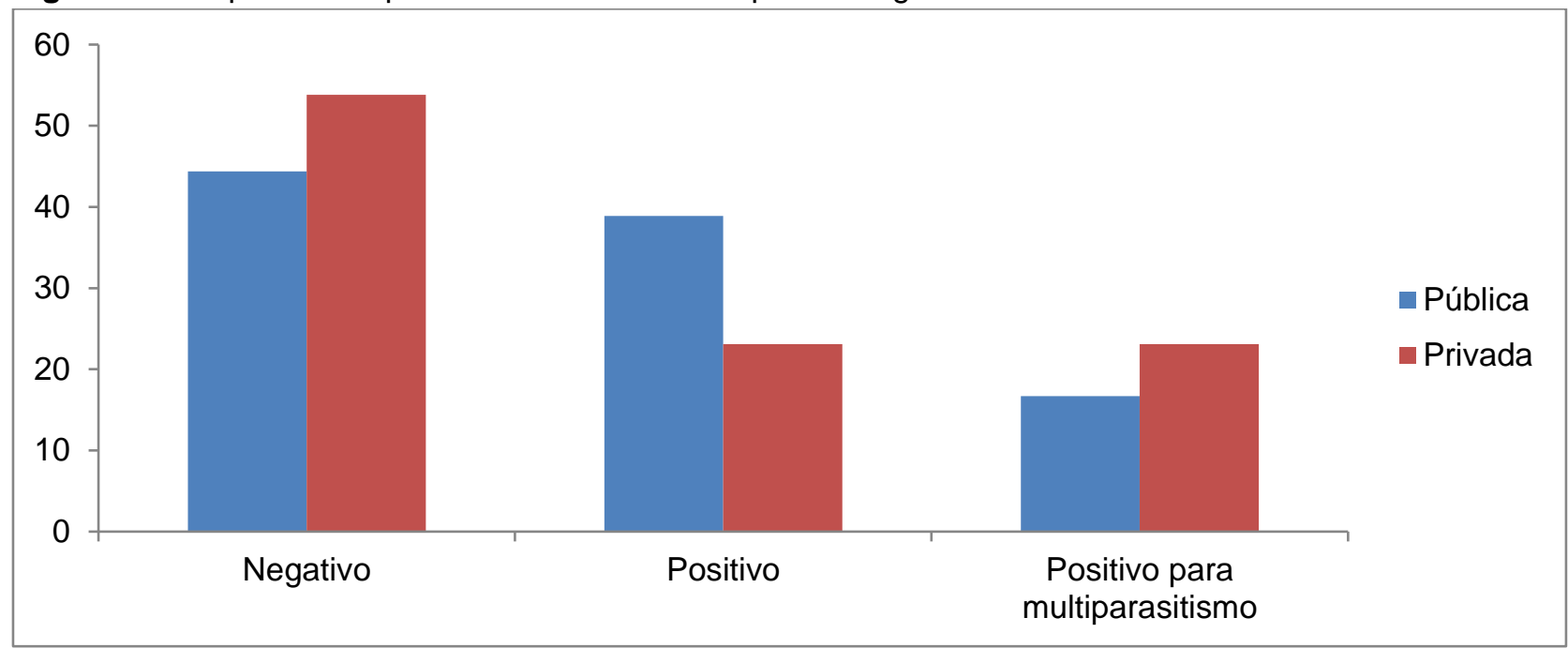

Fonte: Cruz JS, et al., 2019.

Conforme observado na Figura 2, a escola privada uma prevalência de cistos de Giardia Lambria (23\%), Ascaris Lumbricoides infértil (15\%), dois ou mais parasitas (8\%) sendo estes, Ascaris Lumbricoides infértil com cistos de Giardia Lambria, cisto de Giardia Lambria. com cistos de Endolimax nana ou Ascaris Lumbricoides infértil com cistos de Endolimax nana. Na escola pública os resultados dos exames parasitológicos de fezes apontaram uma prevalência de cistos de Giardia Lambria (22\%), Ascaris Lumbricoides infértil (17\%), Larva Strongyloides Stercolaris filarióide (6\%), dois ou mais parasitas (11\%) sendo estes Ascaris Lumbricoides infértil com cistos de Giardia Lambria.

Figura 2 - Resultado do exame parasitológico de fezes.

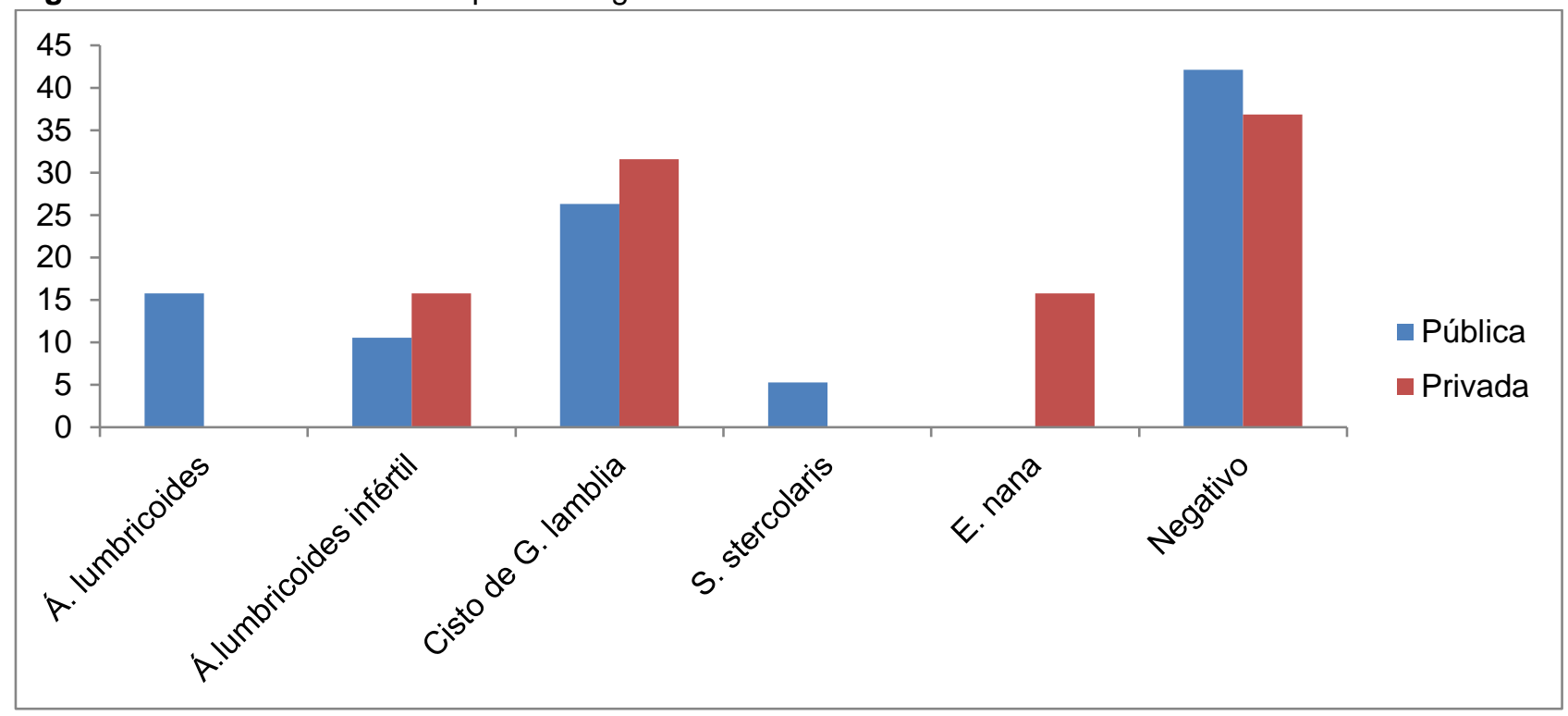

Fonte: Cruz JS, et al., 2019. 


\section{DISCUSSÃO}

Como observado nos resultados, a frequência de água filtrada na escola pública $(94,4 \%)$ é maior que a escola particular (73,0\%). Já em relação à água clorada a escola pública apresenta um índice menor (5,6\%) que a escola particular (26,9\%). Segundo, Vargas MI (2015), o tratamento de água, construção de redes de esgoto e tratamento de água, são medidas de saneamento básico que ajudam a prevenir doenças hídricas vinculadas a poluição fecal.

$\mathrm{Na}$ análise do questionário verificou-se $38,8 \%$ dos alunos da escola pública apresentaram animais em suas residências comparados à escola privada que apresentou 15,3\%. Blazius RD, et al. (2005), afirma que o parasitismo intestinal de animais domésticos, como cães e gatos têm grande importância em saúde pública, pois muitos destes parasitos podem infectar o homem. Animais domésticos quando interdomiciliados devem ser assistidos frequentemente por médico veterinário, pois são agentes de várias zoonoses e desempenham um papel importante na disseminação de algumas espécies de parasitos.

Diante destes resultados observa-se que o parasita com maior prevalência encontrado nas duas escolas foi de cistos de Giardia Lambria. Pesquisas realizadas comprovam que, no Brasil, a prevalência de giardíase é de $4 \%$ a $30 \%$. De acordo com estudo realizado em 2013 com crianças de 3 (três) a 7 (sete) anos são mais vulneráveis à estas infecções, devido a hábitos de higiene inadequados, perfazendo que, em seu estudo, teve um índice de 23, \% de Giárdia lamblia (BORGES WF, et al., 2011). A giardíase é responsável por cerca de 280 milhões de infecções por ano em esfera âmbito mundial (BERNE et al., 2014), uma ocorrência de $2 \%$ a $7 \%$ nos países desenvolvidos e de $20 \%$ a 30\% nos países em desenvolvimento (KAMEL D, et al., 2013).

Esse alto índice de infeção por G. Lambria indica as condições socioeconômicas pelas as quais estas crianças vivem como este protozoário apresenta uma via de transmissão fecal-oral, a higiene pessoal e ambiental é de extrema importância na fonte de infecção (ARRUDA AAR, et al., 2008). Após serem entregues os laudos com os resultados positivos para os pais ou responsáveis, os casos de giardíase foram tratados com metronidazol, na dose de $20 \mathrm{mg} / \mathrm{kg} /$ dia por um período de sete dias com orientação médica.

A prevalência por $A$. Lumbricoides neste estudo é inferior quando comparada a infecção causada por $G$. Lambria. Este helminto é considerado um dos mais prevalentes em seres humanos. Uma alta prevalência de ascaríase é considerada indicativa de saneamento básico inadequado como a ingestão de alimentos e água contaminada no contado com material subungueal ou com o solo contaminado, seja através do hábito de lavar as mãos e objetos a boca, seja com a prática de geofagia e, raramente, através da inalação ou deglutição de ovos presentes em secreção respiratória contaminada (OLIVEIRA JLL, 2013).

Em vista disso, parasitoses intestinais, distingue-se a ascaridíase, verminose de maior domínio no mundo causada através de nematóide Ascaris lumbricoides. Em 2008, a Organização Mundial de Saúde (OMS) estabeleceu que mais de 980 milhões de pessoas no mundo estariam parasitadas por esse agente (SILVA JRCB e BARBOSA TJP, 2011). O tratamento foi realizado após o resultado positivo do laudo, sob orientação médica. Albendazol $400 \mathrm{mg}$ em dose única. Mebendazol $100 \mathrm{mg}, 2$ vezes ao dia, durante 3 dias consecutivos.

Foi observada uma baixa prevalência de Larva Strongyloides Stercolaris filarióide (6\%) no resultado obtido na escola pública. Em estudo desenvolvido na área metropolitana do Rio de Janeiro, de abril de 2012 a fevereiro de 2015, em uma população de 3.245 indivíduos, de ambos os sexos, encontrou-se $4,3 \%$ de infecções causadas pelo $S$. stercoralis. Segundo Rey, a forma de contaminação mais comum é a penetração ativa da larva filarióide na pele. Outra forma de contaminação menos comum é a via digestiva, por meio da ingestão de água contaminada com larvas infectantes (BRANDELEROA E, et al., 2019).

Observa-se que das crianças pesquisadas contaminadas com enteroparasitas, a minoria apresentou resultado positivo para cistos de Endolimax nana. Este protozoário apesar de não patogênico revela as condições insalubres de higiene, tanto pessoal como ambiental, indicando principalmente a transmissão fecal oral (FERREIRA DS e VIEIRA GO, 2006). O tratamento foi realizado após o resultado positivo do laudo, Secnidazol (1 $1^{a}$ opção) via oral - não recomendado para gestantes (1 Trimestre) e lactentes, Metronidazol via oral por 5 dias, Tinidazol via oral, durante 2 dias, para formas intestinais. 


\section{CONCLUSÃO}

A prevalência de parasitoses intestinais é altamente elevada em locais nos quais as condições de saneamento básico são insatisfatórias ou inexistentes. Desta forma há uma carência de programas educacionais para conscientizar a população por meio de intervenções de educação em saúde, assim como para a realização de exames parasitológicos de fezes uma vez que se torna bastante importante para se descobrir a presença de algum parasita e assim ter o seu devido tratamento. Sendo assim pode-se concluir com base nos resultados obtidos no presente estudo que a presença de enteroparasitoses nas crianças analisadas foi baixa. Este baixo índice pode estar relacionado a fatores como o consumo de água tratada e a presença de rede de esgoto.

\section{REFERÊNCIAS}

1. ARRUDA AAR, et al. Prevalência de giardíase em crianças e seus cães da periferia urbana de Lages, Santa Catarina. Revista da FZVA, 2008; 15(2): 126-134.

2. BERNE AC, et al. Giardia lamblia: Diagnóstico com o emprego de métodos microscópicos e Enzyme-linked Immunosorbent Assay (ELISA). Revista de Patologia Tropical. 2014; 43(4): 412-419.

3. BLAZIUS RD, et al. Ocorrência de protozoários e helmintos em amostras de fezes de cães errantes da Cidade de Itapema, Santa Catarina. Revista da Sociedade Brasileira de Medicina Tropical, 2005; 38(1): 73-74.

4. BELLIN M, GRAZZIOTIN NA. Prevalência de parasitos intestinais no município de Sanandauva/RS. Newslab, 2011; 18(104): 116-122.

5. BORGES WF, MARCIANO FM, OLIVEIRA HB. Parasitos intestinais: elevada prevalência de Giardia lamblia em pacientes atendidos pelo serviço público de saúde da região sudeste de Goiás, Brasil. Revista de patologia tropical, 2011; 40(2): 149-157.

6. BRANDELEROA E, et al. Hemorragia digestiva ocasional em criança por estrongiloidíase: importância do exame parasitológico. Revista Paulista de Pediatria, 2019; 37(1): 121-125.

7. FERNANDES S, et al. Protocolo de parasitoses intestinais. Acta Pediátrica Portuguesa, 2011; 43(1): 34-40.

8. FERREIRA DS, VIEIRA GO. Frequência de enteroparasitas na população atendida pelo laboratório de analíses clínicas Dr. Emmerson Luiz da Costa. Saúde e Ambiente em revista, 2006; 1(2): 70-75.

9. LODO M, et al. Prevalência de enteroparasitas em município do interior paulista. Revista Brasileira de Crescimento e Desenvolvimento Humano, 2010; 20(3); 769-777.

10. KAMEL D, et al. Diagnostic potential of target Giardia lamblia specific antigen for detection of human giardiasis using coproantigen sandwich ELISA. World Journal of Medical Sciences, 2013; 9(2): 113-122.

11. MACCHIONI F, et al. Dramatic Decrease in Prevalence of Soil-Transmitted Helminths and New Insights into Intestinal Protozoa in Children Living in the Chaco Region, Bolivia. Revisit the American Journal of Tropical medicine and Hygiene, 2015; 92(4): 794-796.

12. OLIVEIRA JLL. Parasitoses intestinais: o ensino como ferramenta principal na minimização destas patologias. 2013. Dissertação de mestrado (Ensino em Ciências da Saúde e do Meio Ambiente) - Centro Universitário de Volta Redonda, Volta Redonda, 2013, 78p.

13. SANTOS SM, et al. Estratégias didáticas para abordagem da Enterobiose na educação básica. In: $X$ Encontro Nacional de Pesquisa em Educação em Ciências, 2015, Águas de Lindóia. Anais do X Encontro Nacional de Pesquisa em Educação em Ciências. São Paulo: ENPEC, 2015; 1-8.

14. SILVA JRCB, BARBOSA TJP. Incidência de Parasitoses Intestinais em crianças na faixa etária de 2 a 4 anos atendidas em uma creche pública no município de Macapá-AP. Trabalho de conclusão de curso. Universidade Federal do Amapá, Macapá, 2011.

15. SOUSA CDSS, SOUSA SCS, ALVARES AM. Diretrizes normativas para o saneamento básico no Brasil. Caderno de Geografia, 2015; 25(43): 102-115.

16. VARGAS MI. Plano de ação para redução de parasitoses intestinais em pacientes atendidos no centro de saúde São Francisco, município Cariacica, ES. Monografia (Especialização em Saúde da Família). Universidade Aberta do SUS, Espírito Santo, 2015, 17p. 